\title{
MEMOIRS OF THE AMERICAN MATHEMATICAL SOCIETY
}

The American Mathematical Society will issue in the near future the first number of a new medium of publication to be known as the Memoirs of the American Mathematical Society. This new series is designed particularly for long papers and for groups of cognate papers. The Memoirs are to be under the supervision of the Editorial Committee of the Transactions of the American Mathematical Society and to be subject to the same standards for acceptance of manuscripts as those now used for the Transactions.

The Memoirs are to be printed by the photo-offset process from copy fully prepared by the authors, following instructions formulated in a manual. A committee consisting of B. P. Gill (Chairman), A. W. Tucker, G. T. Whyburn, and J. W. T. Youngs is now considering the various problems concerning the manual of instructions. Until this manual appears, authors desiring to submit manuscripts for the new series are advised to consult this committee.

The Memoirs are to be nonperiodical, but serial, publications; in this respect, they are like the volumes in Mathematical Surveys or the Colloquium Publications. They will be issued and sold separately. As they are ready for distribution, members will receive suitable announcements and order cards. Individuals and institutions will be given the opportunity to place standing orders for all numbers in the series. As the manuscripts are to be fully prepared by the authors for photo-offsetting, there will be no expense to the Society for typesetting. Therefore it is hoped that it will be possible to sell the Memoirs at a low price.

One of the factors which has caused the heavy backlog of papers in the Transactions is the number of very long manuscripts worthy of publication which have been and are being accepted by the Editorial Committee of the Transactions. With the existence of this new series, the editors of the Transactions are setting increasingly higher standards for longer papers and the publication of these papers may be deferred at the discretion of the editors. Papers of more than a specified size are not to be printed in the Transactions, except in unusual cases, but are to be published in the Memoirs. In this manner authors whose papers are accepted for the Memoirs can expect prompt publication. This is a great advantage, both to the author and to mathematics in general, for at the present time there is of ten a period of eighteen months between acceptance and publication. By 
relieving the pressure on the Transactions, the Council hopes that the Bulletin will receive some corresponding relief.

The new series will be inaugurated by the publication of two Memoirs. The first will be entitled Open mappings on locally compact spaces by G. T. Whyburn and the second will contain papers by Jean Dieudonné and L. K. Hua, entitled On automorphisms of the classical groups and Supplement to the preceding paper by Dieudonné, respectively.

J. R. KLINE, Secretary 